\title{
Quantitative Analysis of Correlated Atomic Displacements via Diffuse Electron Scattering
}

\author{
Xi Chen, Georgios Varnavides, Polina Anikeeva and James LeBeau \\ Massachusetts Institute of Technology, Cambridge, Massachusetts, United States
}

Momentum-resolved information on phonons can be inferred from neutron and X-ray scattering experiments by utilizing momentum conservation $[1,2]$. In contrast to Bragg diffraction, thermal diffuse scattering intensities are distributed across reciprocal space and can deviate strongly from the isotropic Einstein model due to their dependence on correlated atomic displacements. Applications of electron diffraction to studies of phonon distribution have been limited by the detector dynamic range and detector sensitivity [3, 4]. Specifically, the intense direct beam and Bragg reflections make it difficult to normalize the intensity of the diffuse background to an absolute intensity scale. Therefore, it is difficult to obtain quantitative information that can be directly compared to theoretical predictions. With the development of new direct electron detectors, such as the Electron Microscopy Pixel Array Detector (EMPAD), which enable capturing single electrons with a dynamic range of 1,000,000:1 [5], detailed quantification of the full electron scatter distribution (diffuse and Bragg) became possible.

In this presentation, we will discuss an approach to capture diffuse scattering and Bragg reflections on an absolute intensity scale using a Si single crystal as a testbed. We will show this can be achieved by shifting the diffraction information across an EMPAD. Even though the detector is only128x128 pixels in size, it can finely sample reciprocal space all the way out to the objective lens bore, as shown in Figure 1. Further, we will briefly discuss the methodology used to reconstruct the final diffraction pattern, particularly the need to account for shift errors introduced by hysteresis. We highlight how the diffuse scattering and the Bragg reflections can be normalized with respect to the incident beam, enabling direct comparison with theory. We will compare the experimental results to computed kinematical and dynamical thermal diffuse scatter distributions taking into account canonical ensembles of phonon displacements obtained ab initio, as shown in Figure 2(b, d). Expanding the analysis, we will discuss the quantification of diffuse scattering as a function of sample thickness as well as a function of scattering angle. Furthermore, we will present a method to remove plasmon scattering from the EMPAD dataset, despite being a pre-filter camera.

Finally, the limitations of the simulation approaches will also be discussed. For example, while kinematical simulations are significantly faster, they are quantitatively incorrect for smaller scattering angles where dynamical scattering dominates. At high scattering angles, however, the kinematical simulations reproduce the experimental diffuse scattering as shown in Figure 2(c,d) [6]. 


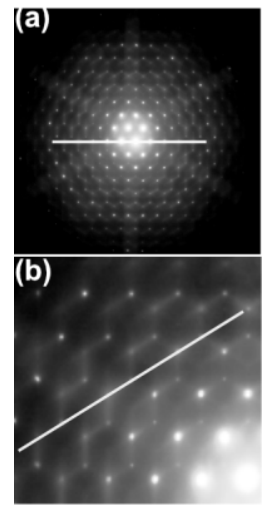

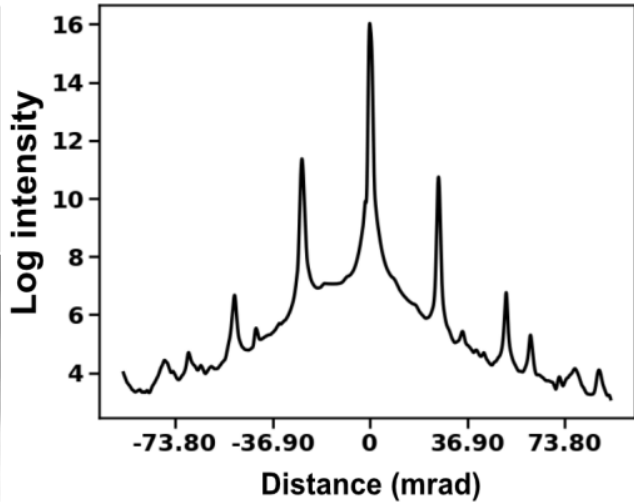

(c)

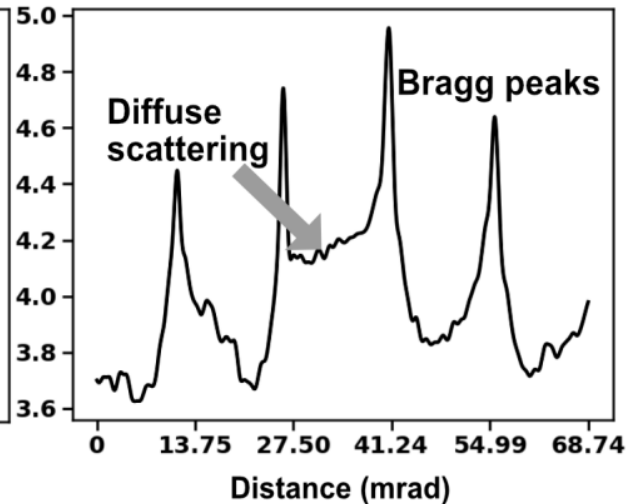

(d)

Figure 1. (a) Finely sampled diffraction pattern of Si oriented along <111> captured with an EMPAD and displayed on a log scale. (b) Subsection of (a) showing the captured details of incoherent scattering simultaneous with the Bragg reflections displayed on a log scale. (c, d) Line profiles from the diffraction pattern highlighting the signal to noise capable of capturing the Bragg reflections and diffuse background.

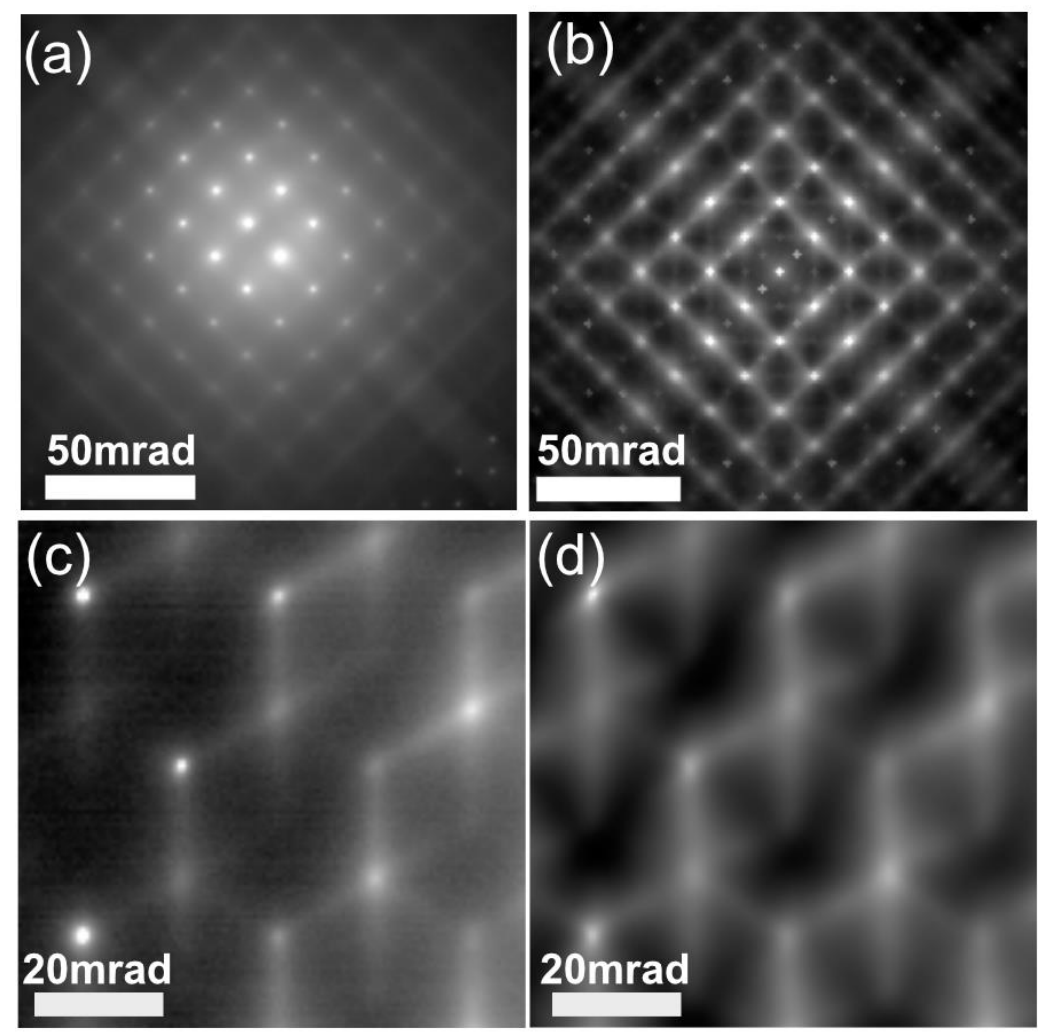

Figure 2. (a) Si diffraction pattern along <100> captured with EMPAD using the diffraction shift methodology. (b) Dynamical simulation of the diffraction pattern of a $32.8 \AA ̊ x 32.8 \AA x 262.4 \AA$ Si supercell oriented along $\langle 100\rangle$. (c) Subsection of a Si diffraction pattern along $\langle 111\rangle$ at high scattering angles and (d) a corresponding kinematical simulation. 


\section{References}

[1] W. Zhou and T. Yildirim, Phys. Rev. B 74 (2006), P180301(R)

[2] Ruqing Xu et al., Z. Kristallogr. 220 (2005), P1009-1016

[3] David A. Muller et al., Ultramicroscopy 86 (2001), p371-380

[4] Renhui Wang et al., Ultramicroscopy 98 (2004), P159-163

[5] MW Tate et al., Microsc. Microanal 22 (2016), P237-249

[6] We acknowledge support for this work from the Air Force Office of Scientific Research (FA9550-171-0225. This work was performed in part at the Analytical Instrumentation Facility (AIF) at North Carolina State University, which is supported by the State of North Carolina and the National Science Foundation (award number ECCS-1542015). The AIF is a member of the North Carolina Research Triangle Nanotechnology Network (RTNN), a site in the National Nanotechnology Coordinated Infrastructure (NNCI). 